\title{
A Priori Error Estimates of Mixed Finite Element Methods for General Linear Hyperbolic Convex Optimal Control Problems
}

\author{
Zuliang Lu ${ }^{1,2}$ and Xiao Huang ${ }^{3}$ \\ ${ }^{1}$ School of Mathematics and Statistics, Chongqing Three Gorges University, Chongqing 404000, China \\ ${ }^{2}$ Laboratory for Applied Mathematics, Beijing Computational Science Research Center, Beijing 100084, China \\ ${ }^{3}$ College of Electronic and Information Engineering, Chongqing Three Gorges University, Chongqing 404000, China
}

Correspondence should be addressed to Zuliang Lu; zulianglux@126.com

Received 21 March 2014; Accepted 16 June 2014; Published 21 July 2014

Academic Editor: Manuel Ruiz-Galan

Copyright (C) 2014 Z. Lu and X. Huang. This is an open access article distributed under the Creative Commons Attribution License, which permits unrestricted use, distribution, and reproduction in any medium, provided the original work is properly cited.

\begin{abstract}
The aim of this work is to investigate the discretization of general linear hyperbolic convex optimal control problems by using the mixed finite element methods. The state and costate are approximated by the $k$ order $(k \geq 0)$ Raviart-Thomas mixed finite elements and the control is approximated by piecewise polynomials of order $k$. By applying the elliptic projection operators and Gronwall's lemma, we derive a priori error estimates of optimal order for both the coupled state and the control approximation.
\end{abstract}

\section{Introduction}

With the advances of scientific computing, optimal control problems are now widely used in multidisciplinary applications such as physics, biology, medicine, engineering design, fluid mechanics, and social-economic systems. The finite element method is undoubtedly the most widely used numerical method in computing optimal control problems. Finite element approximation of a class of elliptic optimal control problems has been studied by Falk in [1]. Then, Alt and Mackenroth in [2] established a priori error estimates for the finite element approximations to state constrained convex parabolic boundary control problems. Finite element approximation of optimal control problems was developed in [3-16], but there are very less published results on this topic for hyperbolic optimal control problems.

Since the pioneering work of Brezzi and Fortin [17], the mixed finite element methods to second order elliptic problems have drawn the attention of many specialists in partial differential equations. Mixed finite elements are appropriate for the state equations in such cases since both the scalar variable and its flux variable can be approximated to the same accuracy. In finite element methods, mixed finite element methods were widely used to approximate flux variables, although there was only very limited research work on analyzing such elements for optimal control problems. More recently, in [9], the authors derived a priori error estimates and superconvergence for bilinear quadratic optimal control problems using mixed finite element methods. A posteriori error analysis of mixed finite element methods for some optimal control problems was addressed in [18, 19]. In [20], the author discussed the semidiscrete mixed finite element methods for quadratic hyperbolic optimal control problems. By using mixed elliptic reconstruction methods, he obtained a posteriori $L^{\infty}\left(L^{2}\right)$-error estimates for both the state and the control approximation.

The purpose of this work is to obtain a priori error estimates of mixed finite element methods for general convex optimal control problems governed by linear hyperbolic partial differential equations. Analogous a priori error estimates of mixed finite element solutions for optimal control problems governed by linear parabolic equations can be found in [21]. However, it does not seem to be straightforward to extend the existing techniques to general optimal control problems involving hyperbolic equations.

For $1 \leq p<\infty$ and $m$ any nonnegative integer, let $W^{m, p}(\Omega)=\left\{v \in L^{p}(\Omega) ; D^{\alpha} v \in L^{p}(\Omega)\right.$ if $|\alpha| \leq$ $m$ \} denote the Sobolev spaces endowed with the norm $\|v\|_{m, p}^{p}=\sum_{|\alpha| \leq m}\left\|D^{\alpha} v\right\|_{L^{p}(\Omega)}^{p^{p}}$ and the seminorm $|v|_{m, p}^{p}=$ $\sum_{|\alpha|=m}\left\|D^{\alpha} v\right\|_{L^{p}(\Omega)}^{p}$. We set $W_{0}^{m, p}(\Omega)=\left\{v \in W^{m, p}(\Omega)\right.$ : $\left.\left.v\right|_{\partial \Omega}=0\right\}$. For $p=2$, we denote $H^{m}(\Omega)=W^{m, 2}(\Omega)$, 
$H_{0}^{m}(\Omega)=W_{0}^{m, 2}(\Omega)$ and $\|\cdot\|_{m}=\|\cdot\|_{m, 2},\|\cdot\|=\|\cdot\|_{0,2} \cdot$ We denote by $L^{s}\left(J ; W^{m, p}(\Omega)\right)$ the Banach space of all $L^{s}$ integrable functions from $J$ to $W^{m, p}(\Omega)$ with norm $\|v\|_{L^{s}\left(J ; W^{m, p}(\Omega)\right)}=$ $\left(\int_{0}^{T}\|v\|_{W^{m, p}(\Omega)}^{s} d t\right)^{1 / s}$ for $s \in[1, \infty),\|v\|_{L^{\infty}\left(J ; W^{m, p}(\Omega)\right)}=$ $\sup _{t \in J}\|v\|_{W^{m, p}(\Omega)}$, and the standard modification for $s=\infty$.

In this paper, we focus our attention on the following general linear hyperbolic convex optimal control problems:

$$
\min _{u \in K \subset U}\left\{\int_{0}^{T}\left(g_{1}(\mathbf{p})+g_{2}(y)+h(u)\right) d t\right\},
$$

subject to the state equations

$$
\begin{aligned}
y_{t t}+\operatorname{div} \mathbf{p} & =f+u, \quad x \in \Omega, t \in J, \\
\mathbf{p} & =-A \nabla y, \quad x \in \Omega, t \in J, \\
\left.y\right|_{\partial \Omega} & =0, \quad t \in J, \\
y(x, 0) & =y_{0}(x), \quad x \in \Omega, \\
y_{t}(x, 0) & =y_{1}(x), \quad x \in \Omega,
\end{aligned}
$$

where the bounded open set $\Omega \subset \mathbb{R}^{2}$ is a convex polygon with the boundary $\partial \Omega, \Omega_{U}$ is a bounded open set in $\mathbb{R}^{2}$ with the Lipschitz boundary $\partial \Omega_{U}, g_{1}, g_{2}$, and $h$ are convex functionals, and $J=[0, T]$. We assume that $K$ is a closed convex set in $U=L^{2}\left(J ; L^{2}\left(\Omega_{U}\right)\right), f \in L^{2}\left(J ; L^{2}(\Omega)\right)$, and $y_{0}, y_{1} \in H^{1}(\Omega)$. Furthermore, we assume the coefficient matrix $A(x)=$ $\left(a_{i, j}(x)\right)_{2 \times 2} \in\left(W^{1, \infty}(\Omega)\right)^{2 \times 2}$ is a symmetric $2 \times 2$-matrix and there is a constant $c>0$ satisfying for any vector $\mathbf{X} \in \mathbb{R}^{2}$, $\mathbf{X}^{\prime} A \mathbf{X} \geq c\|\mathbf{X}\|_{\mathbb{R}^{2}}^{2}$. The set of admissible controls $K$ is defined by

$$
K=\left\{u \in U=L^{2}\left(J ; L^{2}\left(\Omega_{U}\right)\right): \int_{0}^{T} \int_{\Omega_{U}} u d x d t \geq 0\right\} .
$$

The remainder of the paper is organized as follows. In Section 2, we construct the $k$ order Raviart-Thomas mixed finite element approximation for general convex optimal control problems governed by linear hyperbolic equations and briefly state the definitions and properties of some interpolation operators. In Section 3, we derive a priori error estimates of the mixed finite element solutions for the general hyperbolic optimal control problems. Finally, we give the conclusion and the future work in Section 4.

\section{Mixed Methods of Hyperbolic Optimal Control}

We will now describe the mixed finite element discretization of general linear hyperbolic convex optimal control problems (1)-(6). Firstly, we introduce the costate hyperbolic equation,

$$
\begin{array}{r}
z_{t t}(x, t)-\operatorname{div}\left(A\left(\nabla z(x, t)+g_{1}^{\prime}(\mathbf{p}(x, t))\right)\right)=g_{2}^{\prime}(y(x, t)), \\
x \in \Omega,
\end{array}
$$

with the conditions,

$$
\begin{aligned}
& \left.z\right|_{\partial \Omega}=0, \quad t \in J ; \quad z(x, T)=0, \quad x \in \Omega ; \\
& z_{t}(x, T)=0, \quad x \in \Omega .
\end{aligned}
$$

Next, we need the following regularity assumptions for the hyperbolic equations (2) and (8): there exists a constant $C$ such that

$$
\begin{gathered}
\|y\|_{L^{\infty}\left(J ; H^{k+2}(\Omega)\right)}+\left\|y_{t}\right\|_{L^{\infty}\left(J ; H^{k+2}(\Omega)\right)}+\left\|y_{t t}\right\|_{L^{2}\left(J ; H^{k+2}(\Omega)\right)} \leq C \\
\|z\|_{L^{\infty}\left(J ; H^{k+2}(\Omega)\right)}+\left\|z_{t}\right\|_{L^{\infty}\left(J ; H^{k+2}(\Omega)\right)} \leq C \\
\|\mathbf{p}\|_{L^{\infty}\left(J ;\left(H^{k+2}(\Omega)\right)^{2}\right)}+\|\mathbf{q}\|_{L^{\infty}\left(J ;\left(H^{k+2}(\Omega)\right)^{2}\right)} \\
+\left\|\mathbf{q}_{t}\right\|_{L^{\infty}\left(J ;\left(H^{k+1}(\Omega)\right)^{2}\right)} \leq C
\end{gathered}
$$

We will take the state spaces $L^{2}(\mathbf{V})=L^{2}(J ; \mathbf{V})$ and $L^{2}(W)=L^{2}(J ; W)$, where $\mathbf{V}$ and $W$ are defined as follows:

$$
\begin{aligned}
& \mathbf{V}=H(\operatorname{div} ; \Omega)=\left\{\mathbf{v} \in\left(L^{2}(\Omega)\right)^{2}, \operatorname{div} \mathbf{v} \in L^{2}(\Omega)\right\}, \\
& W=L^{2}(\Omega) .
\end{aligned}
$$

The Hilbert space $\mathbf{V}$ is equipped with the following norm:

$$
\|\mathbf{v}\|_{H(\operatorname{div} ; \Omega)}=\left(\|\mathbf{v}\|_{0, \Omega}^{2}+\|\operatorname{div} \mathbf{v}\|_{0, \Omega}^{2}\right)^{1 / 2} .
$$

We recast (1)-(5) as the following weak form: find $(\mathbf{p}, y, u) \in L^{2}(\mathbf{V}) \times L^{2}(W) \times K$ such that

$$
\begin{gathered}
\min _{u \in K}\left\{\int_{0}^{T}\left(g_{1}(\mathbf{p})+g_{2}(y)+h(u)\right) d t\right\}, \\
\left(A^{-1} \mathbf{p}, \mathbf{v}\right)-(y, \operatorname{div} \mathbf{v})=0, \quad \forall \mathbf{v} \in \mathbf{V}, \\
\left(y_{t t}, w\right)+(\operatorname{div} \mathbf{p}, w)=(f+u, w), \quad \forall w \in W, \\
y(x, 0)=y_{0}(x), \quad \forall x \in \Omega, \\
y_{t}(x, 0)=y_{1}(x), \quad \forall x \in \Omega .
\end{gathered}
$$

Hereafter, we assume that $h(u)=\int_{\Omega_{U}} j(u) d x$, where $j(\cdot)$ is a convex continuously differentiable function on $\mathbb{R}$. Then, it is easy to see that $\left(h^{\prime}(u), \mathbf{v}\right)_{U}=\left(j^{\prime}(u), \mathbf{v}\right)_{U}=\int_{\Omega_{U}} j^{\prime}(u) \mathbf{v} d x$.

Taking into account the precious result in $[20,22]$, the optimal control problem (13) has a unique solution (p, $y, u)$, and a triplet $(\mathbf{p}, y, u)$ is the solution of (13) if and only if there 
is a costate $(\mathbf{q}, z) \in L^{2}(\mathbf{V}) \times L^{2}(W)$ such that $(\mathbf{p}, y, \mathbf{q}, z, u)$ satisfies the following optimality conditions:

$$
\begin{aligned}
\left(A^{-1} \mathbf{p}, \mathbf{v}\right)-(y, \operatorname{div} \mathbf{v}) & =0, \quad \forall \mathbf{v} \in \mathbf{V}, \\
\left(y_{t t}, w\right)+(\operatorname{div} \mathbf{p}, w) & =(f+u, w), \quad \forall w \in W, \\
y(x, 0) & =y_{0}(x), \quad \forall x \in \Omega, \\
y_{t}(x, 0) & =y_{1}(x), \quad \forall x \in \Omega, \\
\left(A^{-1} \mathbf{q}, \mathbf{v}\right)-(z, \operatorname{div} \mathbf{v}) & =-\left(g_{1}^{\prime}(\mathbf{p}), \mathbf{v}\right), \quad \forall \mathbf{v} \in \mathbf{V}, \\
\left(z_{t t}, w\right)+(\operatorname{div} \mathbf{q}, w) & =\left(g_{2}^{\prime}(y), w\right), \quad \forall w \in W, \\
z(x, T) & =0, \quad \forall x \in \Omega, \\
z_{t}(x, T) & =0, \quad \forall x \in \Omega, \\
\int_{0}^{T}\left(j^{\prime}(u)+z, \tilde{u}-u\right)_{U} d t & \geq 0, \quad \forall \tilde{u} \in K,
\end{aligned}
$$

where $(\cdot, \cdot)_{U}$ is the inner product of $U$ and $g_{1}^{\prime}, g_{2}^{\prime}$, and $j^{\prime}$ are the derivatives of $g_{1}, g_{2}$, and $j$. For simplification, the product $(\cdot, \cdot)_{U}$ will be denoted by $(\cdot, \cdot)$.

For ease of exposition, we will assume that $\Omega$ and $\Omega_{U}$ are both polygons. Let $\mathscr{T}_{h}$ and $\mathscr{T}_{h}\left(\Omega_{U}\right)$ be regular triangulations or rectangulations of $\Omega$ and $\Omega_{U}$, respectively. They are assumed to satisfy the angle condition which means that there is a positive constant $C$ such that, for all $\tau \in \mathscr{T}_{h}\left(\tau_{U} \in\right.$ $\left.\mathscr{T}_{h}\left(\Omega_{U}\right)\right), C^{-1} h_{\tau}^{2} \leq|\tau| \leq C h_{\tau}^{2}, C^{-1} h_{\tau_{U}}^{2} \leq\left|\tau_{U}\right| \leq C h_{\tau_{U}}^{2}$, where $|\tau|$ is the area of $\tau,\left|\tau_{U}\right|$ is the area of $\tau_{U}, h_{\tau}$ is the diameter of $\tau$, and $h_{\tau_{U}}$ is the diameter of $\tau_{U}$. Let $h=\max h_{\tau}\left(h_{U}=\right.$ $\left.\max h_{\tau_{U}}\right)$. In addition, $C$ or $c$ denotes a general positive constant independent of $h$.

Let $\mathbf{V}_{h} \times W_{h} \subset \mathbf{V} \times W$ denote the order $k$ RaviartThomas space [23] associated with the triangulations or rectangulations $\mathscr{T}_{h}$ of $\Omega$. $P_{k}$ denotes the space of polynomials of total degree at most $k$ and $Q_{m, n}$ indicates the space of polynomials of degree no more than $m$ and $n$ in $x$ and $y$, respectively. If $\tau$ is a triangle, $\mathbf{V}(\tau)=\left\{\mathbf{v} \in P_{k}^{2}(\tau)+x \cdot P_{k}(\tau)\right\}$, and if $\tau$ is a rectangle, $\mathbf{V}(\tau)=\left\{\mathbf{v} \in Q_{k+1, k}(\tau) \times Q_{k, k+1}(\tau)\right\}$, $W(\tau)=P_{k}(\tau)$. We define

$$
\begin{aligned}
\mathbf{V}_{h} & :=\left\{\mathbf{v}_{h} \in \mathbf{V}: \forall \tau \in \mathscr{T}_{h},\left.\mathbf{v}_{h}\right|_{\tau} \in \mathbf{V}(\tau)\right\}, \\
W_{h} & :=\left\{w_{h} \in W: \forall \tau \in \mathscr{T}_{h},\left.w_{h}\right|_{\tau} \in W(\tau)\right\}, \\
U_{h} & :=\left\{\widetilde{u}_{h} \in U: \forall \tau \in \mathscr{T}_{h}\left(\Omega_{U}\right),\left.\widetilde{u}_{h}\right|_{\tau} \in W(\tau)\right\} .
\end{aligned}
$$

By the definition of finite element subspace, the mixed finite element discretization of (13) is as follows: compute $\left(\mathbf{p}_{h}, y_{h}, u_{h}\right) \in L^{2}\left(\mathbf{V}_{h}\right) \times L^{2}\left(W_{h}\right) \times K_{h}$ such that

$$
\begin{gathered}
\min _{u_{h} \in K_{h} \subset U_{h}}\left\{\int_{0}^{T}\left(g_{1}\left(\mathbf{p}_{h}\right)+g_{2}\left(y_{h}\right)+h\left(u_{h}\right)\right) d t\right\}, \\
\left(A^{-1} \mathbf{p}_{h}, \mathbf{v}_{h}\right)-\left(y_{h}, \operatorname{div} \mathbf{v}_{h}\right)=0, \quad \forall v_{h} \in V_{h}, \\
\left(y_{h t t}, w_{h}\right)+\left(\operatorname{div} \mathbf{p}_{h}, w_{h}\right)=\left(f+u_{h}, w_{h}\right), \quad \forall w_{h} \in W_{h}, \\
y_{h}(x, 0)=y_{0}^{h}(x), \quad \forall x \in \Omega, \\
y_{h t}(x, 0)=y_{1}^{h}(x), \quad \forall x \in \Omega,
\end{gathered}
$$

where $K_{h}=U_{h} \cap K$ and $y_{0}^{h}(x)$ and $y_{1}^{h}(x) \in W_{h}$ are two finite element approximations of $y_{0}(x)$ and $y_{1}(x)$.

It is well known (see, e.g., [7, 20]) that the optimal control problem (24) again has a unique solution $\left(\mathbf{p}_{h}, y_{h}, u_{h}\right)$ and that a triplet $\left(\mathbf{p}_{h}, y_{h}, u_{h}\right)$ is the solution of (24) if and only if there is a costate $\left(\mathbf{q}_{h}, z_{h}\right) \in L^{2}\left(\mathbf{V}_{h}\right) \times L^{2}\left(W_{h}\right)$ such that $\left(\mathbf{p}_{h}, y_{h}, \mathbf{q}_{h}, z_{h}, u_{h}\right)$ satisfies the following optimality conditions:

$$
\begin{aligned}
\left(A^{-1} \mathbf{p}_{h}, \mathbf{v}\right)-\left(y_{h}, \operatorname{div} v\right) & =0, \quad \forall \mathbf{v} \in \mathbf{V}_{h}, \\
\left(y_{h t t}, w\right)+\left(\operatorname{div} \mathbf{p}_{h}, w\right) & =\left(f+u_{h}, w\right), \quad \forall w \in W_{h} \\
y_{h}(x, 0) & =y_{0}^{h}(x), \quad \forall x \in \Omega \\
y_{h t}(x, 0) & =y_{1}^{h}(x), \quad \forall x \in \Omega, \\
\left(A^{-1} \mathbf{q}_{h}, \mathbf{v}\right)-\left(z_{h}, \operatorname{div} \mathbf{v}\right) & =-\left(g_{1}^{\prime}\left(\mathbf{p}_{h}\right), \mathbf{v}\right), \quad \forall \mathbf{v} \in \mathbf{V}_{h}, \\
\left(z_{h t t}, w\right)+\left(\operatorname{div} \mathbf{q}_{h}, w\right) & =\left(g_{2}^{\prime}\left(y_{h}\right), w\right), \quad \forall w \in W_{h} \\
z_{h}(x, T) & =0, \quad \forall x \in \Omega \\
z_{h t}(x, T) & =0, \quad \forall x \in \Omega \\
\int_{0}^{T}\left(j^{\prime}\left(u_{h}\right)+z_{h}, \tilde{u}-u_{h}\right)_{U} d t & \geq 0, \quad \forall \tilde{u} \in K_{h} .
\end{aligned}
$$

Let $P_{h}: W \rightarrow W_{h}$ be the orthogonal $L^{2}(\Omega)$-projection into $W_{h}$ defined by

$$
\left(P_{h} w-w, \chi\right)=0, \quad w \in W, \chi \in W_{h},
$$

which satisfies

$$
\left\|P_{h} w-w\right\|_{0, q} \leq C\|w\|_{s, q} h^{s}
$$

$0 \leq s \leq k+1, \quad$ if $w \in W \cap W^{s, q}(\Omega)$,

$$
\begin{gathered}
\left\|P_{h} w-w\right\|_{-r} \leq C\|w\|_{s} h^{r+s}, \\
0 \leq r, \quad s \leq k+1, \quad \text { if } w \in H^{s}(\Omega), \\
\left(\operatorname{div} \mathbf{v}, w-P_{h} w\right)=0, \quad w \in W, \mathbf{v} \in \mathbf{V}_{h} .
\end{gathered}
$$

Let $\pi_{h}: \mathbf{V} \rightarrow \mathbf{V}_{h}$ be the Raviart-Thomas projection [24], which satisfies

$$
\begin{gathered}
\left(\operatorname{div}\left(\pi_{h} \mathbf{v}-\mathbf{v}\right), w_{h}\right)=0, \quad \mathbf{v} \in \mathbf{V}, w_{h} \in W_{h}, \\
\left\|\pi_{h} \mathbf{v}-\mathbf{v}\right\|_{0, q} \leq C\|\mathbf{v}\|_{s, q} h^{s}, \\
\frac{1}{q}<s \leq k+1, \quad \text { if } \mathbf{v} \in \mathbf{V} \cap W^{s, q}(\Omega)^{2}, \\
\left\|\operatorname{div}\left(\pi_{h} \mathbf{v}-\mathbf{v}\right)\right\|_{0} \leq C\|\operatorname{div} \mathbf{v}\|_{s} h^{s}, \\
0 \leq s \leq k+1, \quad \text { if } \mathbf{v} \in \mathbf{V} \cap H^{s}(\operatorname{div} ; \Omega) .
\end{gathered}
$$


We have the commuting diagram property

$$
\operatorname{div} \circ \pi_{h}=P_{h} \circ \operatorname{div}: \mathbf{V} \longrightarrow W_{h}, \quad \operatorname{div}\left(I-\pi_{h}\right) \mathbf{V} \perp W_{h},
$$

where $I$ denotes identity matrix. We point out $\left(\pi_{h} \mathbf{v}\right)_{t}=\pi_{h} \mathbf{v}_{t}$ and $\left(P_{h} w\right)_{t}=P_{h} w_{t}$

In the rest of the paper, we will use some intermediate variables. For any control function $\widetilde{u} \in K$, we first define the state solution $(\mathbf{p}(\widetilde{u}), y(\widetilde{u}), \mathbf{q}(\widetilde{u}), z(\widetilde{u}))$ associated with $\widetilde{u}$ that satisfies

$$
\begin{gathered}
\left(A^{-1} \mathbf{p}(\widetilde{u}), \mathbf{v}\right)-(y(\widetilde{u}), \operatorname{div} \mathbf{v})=0, \quad \forall \mathbf{v} \in \mathbf{V}, \\
\left(y_{t t}(\widetilde{u}), w\right)+(\operatorname{div} \mathbf{p}(\widetilde{u}), w)=(f+\widetilde{u}, w), \quad \forall w \in W \\
y(\widetilde{u})(x, 0)=y_{0}(x), \quad \forall x \in \Omega, \\
y_{t}(\widetilde{u})(x, 0)=y_{1}(x), \quad \forall x \in \Omega, \\
\left(A^{-1} \mathbf{q}(\widetilde{u}), \mathbf{v}\right)-(z(\widetilde{u}), \operatorname{div} \mathbf{v})=-\left(g_{1}^{\prime}(\mathbf{p}(\widetilde{u})), \mathbf{v}\right) \\
\left(z_{t t}(\widetilde{u}), w\right)+(\operatorname{div} \mathbf{q}(\widetilde{u}), w)=\left(g_{2}^{\prime}(y(\widetilde{u})), w\right) \\
z(\widetilde{u})(x, T)=0, \quad \forall x \in \Omega, \\
z_{t}(\widetilde{u})(x, T)=0, \quad \forall x \in \Omega
\end{gathered}
$$

Correspondingly, we define the discrete state solution $\left(\mathbf{p}_{h}(\widetilde{u}), y_{h}(\widetilde{u}), \mathbf{q}_{h}(\widetilde{u}), z_{h}(\widetilde{u})\right)$ associated with $\widetilde{u} \in K$ that satisfies

$$
\begin{aligned}
\left(A^{-1} \mathbf{p}_{h}(\widetilde{u}), \mathbf{v}\right)-\left(y_{h}(\widetilde{u}), \operatorname{div} \mathbf{v}\right) & =0, \quad \forall \mathbf{v} \in \mathbf{V}_{h}, \\
\left(y_{h t t}(\widetilde{u}), w\right)+\left(\operatorname{div} \mathbf{p}_{h}(\widetilde{u}), w\right) & =(f+\widetilde{u}, w), \quad \forall w \in W_{h},
\end{aligned}
$$$$
y_{h}(\widetilde{u})(x, 0)=y_{0}^{h}(x), \quad \forall x \in \Omega,
$$$$
y_{h t}(\widetilde{u})(x, 0)=y_{1}^{h}(x), \quad \forall x \in \Omega,
$$

$$
\left(A^{-1} \mathbf{q}_{h}(\widetilde{u}), \mathbf{v}\right)-\left(z_{h}(\widetilde{u}), \operatorname{div} \mathbf{v}\right)=-\left(g_{1}^{\prime}\left(\mathbf{p}_{h}(\widetilde{u})\right), \mathbf{v}\right),
$$$$
\forall \mathbf{v} \in \mathbf{V}_{h}
$$

$$
\begin{array}{r}
\left(z_{h t t}(\widetilde{u}), w\right)+\left(\operatorname{div} \mathbf{q}_{h}(\widetilde{u}), w\right)=\left(g_{2}^{\prime}\left(y_{h}(\widetilde{u})\right), w\right), \\
\forall w \in W_{h}, \\
z_{h}(\widetilde{u})(x, T)=0, \quad \forall x \in \Omega, \\
z_{h t}(\widetilde{u})(x, T)=0, \quad \forall x \in \Omega .
\end{array}
$$$$
\forall w \in W_{h},
$$

Thus, as we defined, the exact solution and its approximation can be written in the following way:

$$
\begin{aligned}
(\mathbf{p}, y, \mathbf{q}, z) & =(\mathbf{p}(u), y(u), \mathbf{q}(u), z(u)), \\
\left(\mathbf{p}_{h}, y_{h}, \mathbf{q}_{h}, z_{h}\right) & =\left(\mathbf{p}_{h}\left(u_{h}\right), y_{h}\left(u_{h}\right), \mathbf{q}_{h}\left(u_{h}\right), z_{h}\left(u_{h}\right)\right) .
\end{aligned}
$$

In the following, we further assume that $g_{1}^{\prime}, g_{2}^{\prime}$, and $j^{\prime}$ are locally Lipschitz continuous, that $g_{1}^{\prime \prime}(\cdot)$ and $g_{2}^{\prime \prime}(\cdot)$ are bound functions on $\left(L^{2}(\Omega)\right)^{2}$ and $L^{2}(\Omega)$, and that there is a $c>0$ such that

$$
\left(j^{\prime}(u)-j^{\prime}(v), u-v\right) \geq c\|u-v\|_{L^{2}\left(\Omega_{U}\right)}^{2}, \quad \forall u, v \in L^{2}\left(\Omega_{U}\right) .
$$

For $\varphi \in W_{h}$, we will write

$$
\phi(\varphi)-\phi(\rho)=\tilde{\phi}^{\prime}(\varphi)(\varphi-\rho),
$$

where $\widetilde{\phi}^{\prime}(\varphi)=\int_{0}^{1} \phi^{\prime}(\varphi+s(\rho-\varphi)) d s$ is bounded function in $\bar{\Omega}[25]$.

\section{A Priori Error Estimates}

Now we will construct an analogue of the family of elliptic projection operators defined by Wheeler [26] in her thesis. Let $(\mathbf{p}, y, \mathbf{q}, z)$ be the solution of (14)-(18). Then, define the elliptic projection of $(\mathbf{p}, y, \mathbf{q}, z)$ to be $(P, Y, Q, Z)$ by the following relations:

$$
\begin{aligned}
\left(A^{-1} P, \mathbf{v}_{h}\right)-\left(Y, \operatorname{div} \mathbf{v}_{h}\right) & =0, \quad \forall \mathbf{v}_{h} \in \mathbf{V}_{h}, \\
\left(\operatorname{div} P, w_{h}\right) & =\left(f+u-y_{t t}, w_{h}\right), \quad \forall w_{h} \in W_{h}, \\
\left(A^{-1} Q, \mathbf{v}_{h}\right)-\left(Z, \operatorname{div} \mathbf{v}_{h}\right) & =-\left(g_{1}^{\prime}(\mathbf{p}), \mathbf{v}_{h}\right), \quad \forall \mathbf{v}_{h} \in \mathbf{V}_{h},
\end{aligned}
$$

$$
\left(\operatorname{div} Q, w_{h}\right)=\left(g_{2}^{\prime}(y)-z_{t t}, w_{h}\right), \quad \forall w_{h} \in W_{h}
$$

where we assume that $Z(x, T)=Z_{t}(x, T)=0$.

Let

$$
\begin{aligned}
\tau_{1}=y-Y, & \sigma_{1}=\mathbf{p}-P, \\
\tau_{2}=z-Z, & \sigma_{2}=\mathbf{q}-Q .
\end{aligned}
$$

From (14)-(18) and (61)-(64), we can easily derive the following error equations:

$$
\begin{aligned}
\left(A^{-1} \sigma_{1}, \mathbf{v}_{h}\right)-\left(\tau_{1}, \operatorname{div} \mathbf{v}_{h}\right)=0, & \forall \mathbf{v}_{h} \in \mathbf{V}_{h}, \\
\left(\operatorname{div} \sigma_{1}, w_{h}\right)=0, & \forall w_{h} \in W_{h}, \\
\left(A^{-1} \sigma_{2}, \mathbf{v}_{h}\right)-\left(\tau_{2}, \operatorname{div} \mathbf{v}_{h}\right)=0, & \forall \mathbf{v}_{h} \in \mathbf{V}_{h}, \\
\left(\operatorname{div} \sigma_{2}, w_{h}\right)=0, & \forall w_{h} \in W_{h} .
\end{aligned}
$$

Estimates for $\tau_{1}, \tau_{2}, \sigma_{1}, \sigma_{2}$ are given in [27] and are presented in Lemma 1 without proof. 
Lemma 1. Assume that the optimal control problems (1)-(5) have a unique solution $(\mathbf{p}, y, u)$. For $t \in J$ and for $h$ sufficiently small, there is a positive constant $C$ independent of $h$ such that

$$
\begin{array}{ll}
\left\|\sigma_{1}\right\|_{0}+\left\|\tau_{1}\right\|_{0} \leq C h^{k+1}\|y\|_{k+2}, & \text { if } y \in H^{k+2}(\Omega), \\
\left\|\sigma_{2}\right\|_{0}+\left\|\tau_{2}\right\|_{0} \leq C h^{k+1}\|y\|_{k+2}, & \text { if } y \in H^{k+2}(\Omega) .
\end{array}
$$

By using Lemma 3 in [22], we can obtain the following technical results.

Lemma 2. Fort $\in J$ and for h sufficiently small, if $y_{t}, y_{t t}, y_{t t t} \in$ $H^{k+2}(\Omega)$, there is a positive constant $C$ independent of $h$ such that

$$
\begin{gathered}
\left\|\sigma_{1 t}\right\|_{0}+\left\|\tau_{1 t}\right\|_{0} \leq C h^{k+1}\left\|y_{t}\right\|_{k+2}, \\
\left\|\sigma_{1 t t}\right\|_{0}+\left\|\tau_{1 t t}\right\|_{0} \leq C h^{k+1}\left\|y_{t t}\right\|_{k+2}, \\
\left\|\sigma_{1 t t t}\right\|_{0}+\left\|\tau_{1 t t t}\right\|_{0} \leq C h^{k+1}\left\|y_{t t t}\right\|_{k+2} .
\end{gathered}
$$

By Theorem 3 in [28], we can establish the following useful result.

Lemma 3. Suppose $v \in L^{2}\left(J ; H^{1}(\Omega)\right) \cap H^{1}\left(J ; H^{1}(\Omega)^{*}\right)$. Then,

$$
v \in C\left(J ; L^{2}(\Omega)\right), \quad \frac{d}{d t}\|v(t)\|_{0}^{2}=2\left(v^{\prime}(t), v(t)\right), \quad \forall t \in J .
$$

Now, we investigate the intermediate error estimates between $(\mathbf{p}, y, \mathbf{q}, z)$ and the intermediate solution $\left(\mathbf{p}_{h}(u)\right.$, $\left.y_{h}(u), \mathbf{q}_{h}(u), z_{h}(u)\right)$. Benefit from the previous results in this section, we only need to estimate $\left\|P-\mathbf{p}_{h}(u)\right\|,\left\|Y-y_{h}(u)\right\|$ and $\left\|P_{h} \mathbf{q}-\mathbf{q}_{h}(u)\right\|,\left\|\pi_{h} z-z_{h}(u)\right\|$.

Let

$$
\begin{array}{cc}
\alpha_{1}=Y-y_{h}(u), & \beta_{1}=P-\mathbf{p}_{h}(u), \\
\alpha_{2}=P_{h} z-z_{h}(u), & \beta_{2}=\pi_{h} \mathbf{q}-\mathbf{q}_{h}(u) .
\end{array}
$$

Lemma 4. Assume that the optimal control problems (1)-(5) have a unique solution (p, $y, u)$ and that $\Omega$ is 2-regular. Assume that the regularity assumptions (10) are valid. There is a positive constant $C>0$, independent of $h$, such that

$$
\begin{gathered}
\left\|P-\mathbf{p}_{h}(u)\right\|_{L^{\infty}\left(J ;\left(L^{2}(\Omega)\right)^{2}\right)}+\left\|Y-y_{h}(u)\right\|_{L^{\infty}\left(J ; L^{2}(\Omega)\right)} \leq C h^{k+1}, \\
\left\|\pi_{h} \mathbf{q}-\mathbf{q}_{h}(u)\right\|_{L^{\infty}\left(J ;\left(L^{2}(\Omega)\right)^{2}\right)}+\left\|P_{h} z-z_{h}(u)\right\|_{L^{\infty}\left(J ; L^{2}(\Omega)\right)} \leq C h^{k+1} .
\end{gathered}
$$

Proof. Firstly, we prove the first inequality (71). From (61)(62) and (50)-(51), we can derive the following error equations:

$$
\begin{gathered}
\left(A^{-1} \beta_{1}, \mathbf{v}\right)-\left(\alpha_{1}, \operatorname{div} \mathbf{v}\right)=0, \quad \forall \mathbf{v} \in \mathbf{V}_{h} \\
\left(\alpha_{1 t t}, w\right)+\left(\operatorname{div} \beta_{1}, w\right)=-\left((y-Y)_{t t}, w\right), \quad \forall w \in W_{h}
\end{gathered}
$$

Differentiating (73) with respect to $t$, we obtain

$$
\left(A^{-1} \beta_{1 t}, \mathbf{v}\right)-\left(\alpha_{1 t}, \operatorname{div} \mathbf{v}\right)=0, \quad \forall \mathbf{v} \in \mathbf{V}_{h}
$$

Taking $t=0$ and $\mathbf{v}=\beta_{1}(0)$ in (75) and choosing $y_{0}^{h}=Y(x, 0)$ and $y_{1}^{h}=Y_{t}(x, 0)$, we can derive that

$$
\alpha_{1}(0)=\alpha_{1 t}(0)=\beta_{1}(0)=\operatorname{div} \beta_{1}(0)=0
$$

Next, taking $t=0$ and $\mathbf{v}=\beta_{1 t}(0)$ in (75) and choosing $y_{0}^{h}=$ $Y(x, 0)$ and $y_{1}^{h}=Y_{t}(x, 0)$, we also find that $\beta_{1 t}(0)=0$. Now, choosing $w=\alpha_{1}$ and $\mathbf{v}=\beta_{1}$ as test functions in (73) and (74), we have

$$
\left\|\alpha_{1 t}\right\|_{L^{\infty}\left(J ; L^{2}(\Omega)\right)}+\left\|\beta_{1}\right\|_{L^{\infty}\left(J ;\left(L^{2}(\Omega)\right)^{2}\right)} \leq C h^{k+1}\left\|y_{t t}\right\|_{L^{2}\left(J ; H^{k+2}(\Omega)\right)} .
$$

From (76), we find that $\alpha_{1}(0)=0$, and then we have

$$
\left\|\alpha_{1}\right\|_{L^{\infty}\left(J ; L^{2}(\Omega)\right)} \leq C\left\|\alpha_{1 t}\right\|_{L^{2}\left(J ; L^{2}(\Omega)\right)} .
$$

Then we obtain (71) from (77), (78), and the triangle inequality.

Furthermore, we prove the second inequality (72). By using (34), subtract (18)-(19) and (46)-(47) to get the following error equations:

$$
\begin{array}{r}
\left(A^{-1} \beta_{2}, \mathbf{v}\right)-\left(\alpha_{2}, \operatorname{div} \mathbf{v}\right) \\
=-\left(A^{-1}\left(\mathbf{q}-\pi_{h} \mathbf{q}\right)+g_{1}^{\prime}(\mathbf{p})-g_{1}^{\prime}\left(\mathbf{p}_{h}(u)\right), \mathbf{v}\right), \\
\forall \mathbf{v} \in \mathbf{V}_{h}, \\
\left(\alpha_{2 t t}, w\right)+\left(\operatorname{div} \beta_{2}, w\right)=\left(g_{2}^{\prime}(y)-g_{2}^{\prime}\left(y_{h}(u)\right), w\right), \\
\forall w \in W_{h} .
\end{array}
$$

Noting that $\alpha_{2}(T)=P_{h} z(T)-z_{h}(u)(T)=0$ and taking $t=T$ in (79), we find that

$$
\begin{array}{r}
\left(A^{-1} \beta_{2}(T), \mathbf{v}\right) \\
=-\left(A^{-1}\left(\mathbf{q}-\pi_{h} \mathbf{q}\right)(T)+g_{1}^{\prime}(\mathbf{p}(T))-g_{1}^{\prime}\left(\mathbf{p}_{h}(u)(T)\right), \mathbf{v}\right), \\
\forall \mathbf{v} \in \mathbf{V}_{h} .
\end{array}
$$


By using Lemma 1 and (77), we can obtain that

$$
\begin{aligned}
& \left\|\beta_{2}(T)\right\| \\
& \leq C\left\|\left(\mathbf{q}-\pi_{h} \mathbf{q}\right)(T)\right\| \\
& +C \| g_{1}^{\prime}(\mathbf{p}(T)) \\
& \quad-g_{1}^{\prime}\left(\mathbf{p}_{h}(u)(T)\right) \| \\
& \leq C\left\|\left(\mathbf{q}-\pi_{h} \mathbf{q}\right)(T)\right\| \\
& +C\left\|\tilde{g}_{1}^{\prime \prime}(\mathbf{p}(T))\left(\left(\mathbf{p}-\mathbf{p}_{h}(u)\right)(T)\right)\right\| \\
& \leq C h^{k+1}\|\mathbf{q}\|_{L^{\infty}\left(J ;\left(H^{k+2}(\Omega)\right)^{2}\right)} \\
& +C\|\mathbf{p}-P\|_{L^{\infty}\left(J ;\left(L^{2}(\Omega)\right)^{2}\right)} \\
& \quad+C\left\|P-\mathbf{p}_{h}(u)\right\|_{L^{\infty}\left(J ;\left(L^{2}(\Omega)\right)^{2}\right)} \\
& \leq C h^{k+1} .
\end{aligned}
$$

Taking $t=0$ and $w=\alpha_{1 t t}(0)$ in (74), since $\operatorname{div} \beta_{1}(0)=0$, we have

$$
\begin{aligned}
\left\|\alpha_{1 t t}(0)\right\|_{L^{\infty}\left(J ; L^{2}(\Omega)\right)} & \leq C\left\|(y-Y)_{t t}(0)\right\|_{L^{\infty}\left(J ; L^{2}(\Omega)\right)} \\
& \leq C h^{k+1}\left\|y_{t t}\right\|_{L^{\infty}\left(J ; H^{k+2}(\Omega)\right)} .
\end{aligned}
$$

Differentiating (75) and (74) with respect to $t$, we obtain

$$
\begin{aligned}
\left(A^{-1} \beta_{1 t t}, \mathbf{v}\right)-\left(\alpha_{1 t t}, \operatorname{div} \mathbf{v}\right) & =0, \quad \forall \mathbf{v} \in \mathbf{V}_{h}, \\
\left(\alpha_{1 t t t}, w\right)+\left(\operatorname{div} \beta_{1 t}, w\right) & =-\left((y-Y)_{t t t}, w\right), \quad \forall w \in W_{h} .
\end{aligned}
$$

Selecting $\mathbf{v}=\beta_{1 t}$ and $w=\alpha_{1 t t}$ as test functions in (84) and (85), respectively, we get

$$
\begin{array}{r}
\left(\alpha_{1 t t t}, \alpha_{1 t t}\right)+\left(A^{-1} \beta_{1 t t}, \beta_{1 t}\right)=-\left((y-Y)_{t t t}, \alpha_{1 t t}\right) \\
\forall w \in W_{h}
\end{array}
$$

Integrating (86) from 0 to $t$, using (83) and the Gronwall's Lemma, we obtain

$$
\begin{aligned}
& \left\|\alpha_{1 t t}\right\|_{L^{\infty}\left(J ; L^{2}(\Omega)\right)}+\left\|\beta_{1 t}\right\|_{L^{\infty}\left(J ;\left(L^{2}(\Omega)\right)^{2}\right)} \\
& \quad \leq C h^{k+1}\left(\left\|y_{t t}\right\|_{L^{\infty}\left(J ; H^{k+2}(\Omega)\right)}+\left\|y_{t t t}\right\|_{L^{2}\left(J ; H^{k+2}(\Omega)\right)}\right) .
\end{aligned}
$$

Differentiating (79) with respect to $t$, we obtain

$$
\begin{aligned}
&\left(A^{-1} \beta_{2 t}, \mathbf{v}\right)-\left(\alpha_{2 t}, \operatorname{div} \mathbf{v}\right) \\
&=-\left(A^{-1}\left(\mathbf{q}-\pi_{h} \mathbf{q}\right)_{t}+g_{1}^{\prime}\left(\mathbf{p}_{t}\right)-g_{1}^{\prime}\left(\mathbf{p}_{h t}(u)\right), \mathbf{v}\right), \\
& \forall \mathbf{v} \in \mathbf{V}_{h} .
\end{aligned}
$$

Now we choose $w=-\alpha_{2 t}$ and $\mathbf{v}=-\beta_{2}$ as test function in (80) and (88), and we have

$$
\begin{aligned}
-\frac{1}{2} & \frac{d}{d t}\left(\left\|\alpha_{2 t}\right\|+\left\|A^{-1 / 2} \beta_{2}\right\|\right)^{2} \\
= & -\left(g_{2}^{\prime}(y)-g_{2}^{\prime}\left(y_{h}(u)\right), \alpha_{2 t}\right) \\
& +\left(A^{-1}\left(\mathbf{q}-\pi_{h} \mathbf{q}\right)_{t}+g_{1}^{\prime}\left(\mathbf{p}_{t}\right)-g_{1}^{\prime}\left(\mathbf{p}_{h t}(u)\right), \beta_{2}\right) \\
= & -\left(\tilde{g}_{2}^{\prime \prime}(y)\left(y-y_{h}(u)\right), \alpha_{2 t}\right) \\
& +\left(A^{-1}\left(\mathbf{q}-\pi_{h} \mathbf{q}\right)_{t}+\tilde{g}_{1}^{\prime \prime}\left(\mathbf{p}_{t}\right)\left(\mathbf{p}-\mathbf{p}_{h}(u)\right)_{t}, \beta_{2}\right) .
\end{aligned}
$$

Then, integrating (89) from $t$ into $T$, using (83) and (87), we obtain

$$
\begin{aligned}
& \left\|\alpha_{2 t}\right\|_{L^{\infty}\left(J ; L^{2}(\Omega)\right)}+\left\|\beta_{2}\right\|_{L^{\infty}\left(J ;\left(L^{2}(\Omega)\right)^{2}\right)} \\
& \leq C\left(\left\|y-y_{h}(u)\right\|_{L^{\infty}\left(J ; L^{2}(\Omega)\right)}+\left\|\mathbf{q}_{t}-\pi_{h} \mathbf{q}_{t}\right\|_{L^{\infty}\left(J ;\left(L^{2}(\Omega)\right)^{2}\right)}\right. \\
& \left.\quad+\left\|\mathbf{p}-\mathbf{p}_{h}(u)\right\|_{L^{\infty}\left(J ;\left(L^{2}(\Omega)\right)^{2}\right)}\right) \\
& \leq C\left(\|y-Y\|_{L^{\infty}\left(J ; L^{2}(\Omega)\right)}+\left\|Y-y_{h}(u)\right\|_{L^{\infty}\left(J ; L^{2}(\Omega)\right)}\right. \\
& \quad+h^{k+1}\left\|\mathbf{q}_{t}\right\|_{L^{\infty}\left(J ;\left(H^{k+1}(\Omega)\right)^{2}\right)}+\|\mathbf{p}-P\|_{L^{\infty}\left(J ;\left(L^{2}(\Omega)\right)^{2}\right)} \\
& \left.\quad+\left\|P-\mathbf{p}_{h}(u)\right\|_{L^{\infty}\left(J ;\left(L^{2}(\Omega)\right)^{2}\right)}\right) \\
& \leq C h^{k+1} .
\end{aligned}
$$

Note that $Z(x, T)=Z_{t}(x, T)=0$; then $\alpha_{2}(T)=0$. Since $\alpha_{2}(t)=\alpha_{2}(t)-\alpha_{2}(T)=-\int_{t}^{T} \alpha_{2 s} d s$, we have

$$
\left\|\alpha_{2}(t)\right\| \leq C\left\|\alpha_{2 t}\right\|_{L^{\infty}\left(J ; L^{2}(\Omega)\right)} .
$$

Then we complete the proof by combining (90), (91), and the triangle inequality.

Using the Lemmas 1 and 4, we can also derive the following error estimates.

Theorem 5. Assume that the optimal control problems (1)-(5) have a unique solution ( $\mathbf{p}, y, u)$ and that $\Omega$ is 2-regular. Assume that the regularity assumptions (10) are valid. There is a positive constant $C>0$, independent of $h$, such that

$$
\begin{gathered}
\left\|\mathbf{p}-\mathbf{p}_{h}(u)\right\|_{L^{\infty}\left(J ;\left(L^{2}(\Omega)\right)^{2}\right)}+\left\|y-y_{h}(u)\right\|_{L^{\infty}\left(J ; L^{2}(\Omega)\right)} \leq C h^{k+1}, \\
\left\|\mathbf{q}-\mathbf{q}_{h}(u)\right\|_{L^{\infty}\left(J ;\left(L^{2}(\Omega)\right)^{2}\right)}+\left\|z-z_{h}(u)\right\|_{L^{\infty}\left(J ; L^{2}(\Omega)\right)} \leq C h^{k+1} .
\end{gathered}
$$


Proof. Combining Lemmas 1 and 4, (35), (39), and the triangle inequality, we obtain that

$$
\begin{aligned}
\| \mathbf{p}- & \mathbf{p}_{h}(u) \|_{L^{\infty}\left(J ;\left(L^{2}(\Omega)\right)^{2}\right)} \\
\leq & \|\mathbf{p}-P\|_{L^{\infty}\left(J ;\left(L^{2}(\Omega)\right)^{2}\right)} \\
& +\left\|P-\mathbf{p}_{h}(u)\right\|_{L^{\infty}\left(J ;\left(L^{2}(\Omega)\right)^{2}\right)} \\
\leq & C h^{k+1}+C h^{k+1} \\
= & C h^{k+1}, \\
\| y- & y_{h}(u) \|_{L^{\infty}\left(J ; L^{2}(\Omega)\right)} \\
\leq & \|y-Y\|_{L^{\infty}\left(J ; L^{2}(\Omega)\right)}+\left\|Y-y_{h}(u)\right\|_{L^{\infty}\left(J ; L^{2}(\Omega)\right)} \\
\leq & C h^{k+1}+C h^{k+1} \\
= & C h^{k+1} .
\end{aligned}
$$

Similarly, we can also obtain that

$$
\begin{aligned}
\| \mathbf{q} & -\mathbf{q}_{h}(u) \|_{L^{\infty}\left(J ;\left(L^{2}(\Omega)\right)^{2}\right)} \\
& \leq\left\|\mathbf{q}-\pi_{h} \mathbf{q}\right\|_{L^{\infty}\left(J ;\left(L^{2}(\Omega)\right)^{2}\right)}+\left\|\pi_{h} \mathbf{q}-\mathbf{q}_{h}(u)\right\|_{L^{\infty}\left(J ;\left(L^{2}(\Omega)\right)^{2}\right)} \\
& \leq C h^{k+1}+C h^{k+1} \\
& =C h^{k+1} \\
\| z & -z_{h}(u) \|_{L^{\infty}\left(J ; L^{2}(\Omega)\right)} \\
& \leq\left\|z-P_{h} z\right\|_{L^{\infty}\left(J ; L^{2}(\Omega)\right)}+\left\|P_{h} z-z_{h}(u)\right\|_{L^{\infty}\left(J ; L^{2}(\Omega)\right)} \\
& \leq C h^{k+1}+C h^{k+1} \\
& =C h^{k+1}
\end{aligned}
$$

This proves (92).

By applying the results we have proved above, we only need to estimate $\left\|\mathbf{p}_{h}(u)-\mathbf{p}_{h}\right\|_{L^{\infty}\left(J ;\left(L^{2}(\Omega)\right)^{2}\right)}, \| y_{h}(u)-$ $y_{h} \|_{L^{\infty}\left(J ; L^{2}(\Omega)\right)} \quad$ and $\quad\left\|\mathbf{q}_{h}(u)-\mathbf{q}_{h}\right\|_{L^{\infty}\left(J ;\left(L^{2}(\Omega)\right)^{2}\right)}, \quad \| z_{h}(u)-$ $z_{h} \|_{L^{\infty}\left(J ; L^{2}(\Omega)\right)}$. For convenience, let

$$
\begin{array}{ll}
e_{1}=y_{h}(u)-y_{h}, & r_{1}=\mathbf{p}_{h}(u)-\mathbf{p}_{h}, \\
e_{2}=z_{h}(u)-z_{h}, & r_{2}=\mathbf{q}_{h}(u)-\mathbf{q}_{h} .
\end{array}
$$

Theorem 6. Let $\left(\mathbf{p}_{h}, y_{h}, \mathbf{q}_{h}, z_{h}, u_{h}\right) \in\left(\mathbf{V}_{h} \times W_{h}\right)^{2} \times U_{h}$ be the solution of (25)-(33) and $\left(\mathbf{p}_{h}(u), y_{h}(u), \mathbf{q}_{h}(u), z_{h}(u)\right) \in$ $(\mathbf{V} \times W)^{2}$ the solution of (50)-(57) with $\tilde{u}=u$. There is a constant $C>0$, independent of $h$, such that

$$
\begin{aligned}
& \left\|\mathbf{p}_{h}(u)-\mathbf{p}_{h}\right\|_{L^{\infty}\left(J ;\left(L^{2}(\Omega)\right)^{2}\right)}+\left\|y_{h}(u)-y_{h}\right\|_{L^{\infty}\left(j ; L^{2}(\Omega)\right)} \\
& \quad \leq C\left\|u-u_{h}\right\|_{L^{2}\left(J ; L^{2}\left(\Omega_{U}\right)\right)} \\
& \quad\left\|\mathbf{q}_{h}(u)-\mathbf{q}_{h}\right\|_{\left.L^{\infty}\left(j ; L^{2}(\Omega)\right)^{2}\right)}+\left\|z_{h}(u)-z_{h}\right\|_{L^{\infty}\left(j ; L^{2}(\Omega)\right)} \\
& \quad \leq C\left\|u-u_{h}\right\|_{L^{2}\left(j ; L^{2}\left(\Omega_{U}\right)\right)}
\end{aligned}
$$

Proof. From (25)-(26) and (50)-(51), we obtain the following error equations:

$$
\begin{aligned}
\left(A^{-1} r_{1}, \mathbf{v}\right)-\left(e_{1}, \operatorname{div} \mathbf{v}\right) & =0, \quad \forall \mathbf{v} \in \mathbf{V}_{h}, \\
\left(e_{1 t t}, w\right)+\left(\operatorname{div} r_{1}, w\right) & =\left(u-u_{h}, w\right), \quad \forall w \in W_{h} .
\end{aligned}
$$

Let $t=0$ and $\mathbf{v}=r_{1}(0)$ in (98); since $e_{1}(0)=0$, we have $r_{1}(0)=0$. We differentiate (98) with respect to $t$, and we derive

$$
\left(A^{-1} r_{1 t}, \mathbf{v}\right)-\left(e_{1 t}, \operatorname{div} v\right)=0, \quad \forall \mathbf{v} \in \mathbf{V}_{h} .
$$

Choose $w=e_{1 t}$ and $\mathbf{v}=r_{1}$ as test functions and add the two relations of (99) and (100); using the Cauchy inequality, we obtain

$$
\frac{1}{2} \frac{d}{d t}\left(\left\|A^{1 / 2} r_{1}\right\|^{2}+\left\|e_{1 t}\right\|^{2}\right) \leq\left\|u-u_{h}\right\|^{2}+\left\|e_{1 t}\right\|^{2} .
$$

Integrating (101) with respect to time from 0 to $t$, we derive

$$
\left\|r_{1}\right\|^{2}+\left\|e_{1 t}\right\|^{2} \leq C \int_{0}^{t}\left\|u-u_{h}\right\|^{2} d s+C \int_{0}^{t}\left\|e_{1 t}\right\|^{2} d s .
$$

By using Gronwall's lemma to (102), we obtain

$$
\left\|r_{1}\right\|_{L^{\infty}\left(J ;\left(L^{2}(\Omega)\right)^{2}\right)}+\left\|e_{1 t}\right\|_{L^{\infty}\left(J ; L^{2}(\Omega)\right)} \leq C\left\|u-u_{h}\right\|_{L^{2}\left(J ; L^{2}\left(\Omega_{U}\right)\right)} .
$$

Since $e_{1}(t)=e_{1}(t)-e_{1}(0)=\int_{0}^{t} e_{1, s} d s$, using (103), we have

$$
\left\|e_{1}\right\| \leq C\left\|e_{1 t}\right\|_{L^{\infty}\left(J ; L^{2}(\Omega)\right)} \leq C\left\|u-u_{h}\right\|_{L^{2}\left(J ; L^{2}\left(\Omega_{U}\right)\right)} .
$$

Then we derive (96).

From (29)-(30) and (54)-(55), we obtain the following error equations:

$$
\left(A^{-1} r_{2}, \mathbf{v}\right)-\left(e_{2}, \operatorname{div} v\right)=-\left(\tilde{g}_{1}^{\prime}\left(\mathbf{p}_{h}(u)\right) r_{1}, \mathbf{v}\right), \quad \forall \mathbf{v} \in \mathbf{V}_{h},
$$

$$
\left(e_{2 t t}, w\right)+\left(\operatorname{div} r_{2}, w\right)=\left(\tilde{g}_{2}^{\prime}\left(y_{h}(u)\right) e_{1}, w\right), \quad \forall w \in W_{h} .
$$

Let $t=T$ and $\mathbf{v}=r_{2}(T)$ in (105); since $e_{2}(T)=0$, we have

$$
\left\|A^{1 / 2} r_{2}(T)\right\| \leq C\left\|\tilde{g}_{1}^{\prime}\left(\mathbf{p}_{h}(u)\right)\right\| \cdot\left\|r_{1}(T)\right\| \leq C\left\|r_{1}(T)\right\| .
$$

Introduce the symbol $\widehat{\varphi}:=\int_{t}^{T} \varphi(s) d s$, let $\widehat{\tilde{g}_{2}^{\prime} e_{1}}=$ $\int_{t}^{T} \tilde{g}_{2}^{\prime}\left(\mathbf{p}_{h}(u)\right) e_{1} d s$, and integrate (105) with respect to time from $t$ to $T$, and we obtain

$$
-\left(e_{2 t}, w\right)+\left(\operatorname{div} \widehat{r}_{2}, w\right)=\left(\widehat{\widetilde{g}_{2}^{\prime} e_{1}}, w\right), \quad \forall w \in W_{h} .
$$

Set $w=e_{2}$ in (108) and $\mathbf{v}=\widehat{r}_{2}$ in (105), note that $r_{2}=-(d /$ $d t) \widehat{r}_{2}$, and then add those equations to derive

$$
-\frac{1}{2} \frac{d}{d t}\left(\left\|e_{2}\right\|^{2}+\left\|A^{1 / 2} \widehat{r}_{2}\right\|^{2}\right)=\left(\widehat{\widetilde{g}_{2}^{\prime} e_{1}}, e_{2}\right)-\left(r_{1}, \widehat{r}_{2}\right),
$$


Integrating (109) with respect to time from $t$ to $T$, using (107) and Yong's inequalities, we get

$$
\begin{aligned}
& \left\|e_{2}\right\|_{L^{\infty}\left(J ; L^{2}(\Omega)\right)} \\
& \quad \leq C\left(\left\|r_{2}(T)\right\|+\left\|e_{1}\right\|_{L^{2}\left(J ; L^{2}(\Omega)\right)}+\left\|r_{1}\right\|_{L^{2}\left(J ;\left(L^{2}(\Omega)\right)^{2}\right)}\right) \\
& \quad \leq C\left(\left\|r_{1}(T)\right\|+\left\|e_{1}\right\|_{L^{2}\left(J ; L^{2}(\Omega)\right)}+\left\|r_{1}\right\|_{L^{2}\left(J ;\left(L^{2}(\Omega)\right)^{2}\right)}\right) \\
& \quad \leq C\left(\left\|e_{1}\right\|_{L^{2}\left(J ; L^{2}(\Omega)\right)}+\left\|r_{1}\right\|_{L^{2}\left(J ;\left(L^{2}(\Omega)\right)^{2}\right)}\right) .
\end{aligned}
$$

Choosing $\mathbf{v}=r_{2}$ and $w=e_{2}$ as test functions in (105) and (106), it is easy to get

$$
\begin{aligned}
& \left\|e_{2 t}\right\|_{L^{\infty}\left(j ; L^{2}(\Omega)\right)}^{2}+\left\|r_{2}\right\|_{L^{\infty}\left(J ;\left(L^{2}(\Omega)\right)^{2}\right)}^{2} \\
& \leq C\left\|e_{1}\right\|_{L^{\infty}\left(J ; L^{2}(\Omega)\right)}^{2}+C\left\|r_{1}\right\|_{L^{\infty}\left(J ;\left(L^{2}(\Omega)\right)^{2}\right)}^{2} \\
& \quad+C \delta\left(\left\|e_{2}\right\|_{L^{\infty}\left(J ; L^{2}(\Omega)\right)}^{2}+\left\|r_{2}\right\|_{L^{\infty}\left(J ;\left(L^{2}(\Omega)\right)^{2}\right)}^{2}\right),
\end{aligned}
$$

where $\delta$ is an arbitrary small positive constant. Namely,

$$
\begin{aligned}
& \left\|e_{2 t}\right\|_{L^{\infty}\left(J ; L^{2}(\Omega)\right)}^{2}+\left\|r_{2}\right\|_{L^{\infty}\left(J ;\left(L^{2}(\Omega)\right)^{2}\right)}^{2} \\
& \quad \leq C\left\|e_{1}\right\|_{L^{\infty}\left(J ; L^{2}(\Omega)\right)}^{2}+C\left\|r_{1}\right\|_{L^{\infty}\left(J ;\left(L^{2}(\Omega)\right)^{2}\right)}^{2} \\
& \quad \leq C\left\|u-u_{h}\right\|_{L^{2}\left(j ; L^{2}\left(\Omega_{U}\right)\right)} .
\end{aligned}
$$

Combining (103)-(104) and (110)-(112), we derive (97).

In the following, we estimate $\left\|u-u_{h}\right\|_{L^{2}\left(J ; L^{2}\left(\Omega_{U}\right)\right)}$ and then obtain the following main result.

Theorem 7. Let $(\mathbf{p}, y, \mathbf{q}, z, u) \in(\mathbf{V} \times W)^{2} \times U$ and $\left(\mathbf{p}_{h}, y_{h}\right.$, $\left.\mathbf{q}_{h}, z_{h}, u_{h}\right) \in\left(\mathbf{V}_{h} \times W_{h}\right)^{2} \times U_{h}$ be the solutions of (14)-(22) and (25)-(33), respectively. Assume that the regularity assumptions (10) and (59) are valid. Furthermore, one assumes that

$$
u \in H^{k+1}\left(\Omega_{U}\right), \quad j^{\prime}(u)+z \in H^{k+1}\left(\Omega_{U}\right) .
$$

Then, one has

$$
\begin{gathered}
\left\|u-u_{h}\right\|_{L^{2}\left(j ; L^{2}\left(\Omega_{U}\right)\right)} \leq C\left(h^{k+1}+h_{U}^{k+1}\right), \\
\left\|\mathbf{p}-\mathbf{p}_{h}\right\|_{L^{\infty}\left(j ;\left(L^{2}(\Omega)\right)^{2}\right)}+\left\|y-y_{h}\right\|_{L^{\infty}\left(J ; L^{2}(\Omega)\right)} \leq C\left(h^{k+1}+h_{U}^{k+1}\right), \\
\left\|\mathbf{q}-\mathbf{q}_{h}\right\|_{L^{\infty}\left(j ;\left(L^{2}(\Omega)\right)^{2}\right)}+\left\|z-z_{h}\right\|_{L^{\infty}\left(J ; L^{2}(\Omega)\right)} \leq C\left(h^{k+1}+h_{U}^{k+1}\right) .
\end{gathered}
$$

Proof. First, in (34), let $\chi=1$, and we have

$$
\int_{\Omega} P_{h} u d x=\int_{\Omega} u d x
$$

Integrating (115) from 0 to $T$, we can obtain that

$$
\int_{0}^{T} \int_{\Omega} P_{h} u d x d t=\int_{0}^{T} \int_{\Omega} u d x d t \geq 0
$$

Therefore, we know that $P_{h} u \in K_{h}$. Now we choose $\widetilde{u}=u_{h}$ in (22) and $\widetilde{u}_{h}=P_{h} u$ in (33) to get that

$$
\begin{array}{r}
\int_{0}^{T}\left(j^{\prime}(u)+z, u_{h}-u\right)_{U} d t \geq 0, \\
\int_{0}^{T}\left(j^{\prime}\left(u_{h}\right)+z_{h}, P_{h} u-u_{h}\right)_{U} d t \geq 0 .
\end{array}
$$

By using (117) and the assumption (59), we have

$$
\begin{aligned}
c \| u- & u_{h} \|_{L^{2}\left(j L^{2}\left(\Omega_{U}\right)\right)}^{x^{2}} \\
\leq & \int_{0}^{T}\left(j^{\prime}(u)-j^{\prime}\left(u_{h}\right), u-u_{h}\right)_{U} d t \\
= & \int_{0}^{T}\left(j^{\prime}(u)+z, u-u_{h}\right)_{U} d t \\
& +\int_{0}^{T}\left(z_{h}(u)-z, u-u_{h}\right)_{U} d t \\
& -\int_{0}^{T}\left(z_{h}(u)-z_{h}, u-u_{h}\right)_{U} d t \\
& -\int_{0}^{T}\left(j^{\prime}\left(u_{h}\right)+z_{h}, u-u_{h}\right)_{U} d t \\
\leq & \int_{0}^{T}\left(z_{h}(u)-z, u-u_{h}\right)_{U} d t \\
& -\int_{0}^{T}\left(z_{h}(u)-z_{h}, u-u_{h}\right)_{U} d t \\
& +\int_{0}^{T}\left(j^{\prime}\left(u_{h}\right)+z_{h}, P_{h} u-u\right)_{U} d t \\
& +\int_{0}^{T}\left(z_{h}(u)-z, u-u_{h}\right)_{U} d t \\
& +\int_{0}^{T}\left(j^{\prime}(u)+z, P_{h} u-u\right)_{U} d t \\
& +\int_{0}^{T}\left(z_{h}(u)-z_{h}, u-u_{h}\right)_{U} d t \\
&
\end{aligned}
$$

From (25)-(33) and (50)-(57), we have

$$
\begin{gathered}
-\int_{0}^{T}\left(z_{h}(u)-z_{h}, u-u_{h}\right)_{U} d t \\
=-\int_{0}^{T}\left(u-u_{h}, e_{2}\right)_{U} d t
\end{gathered}
$$




$$
\begin{aligned}
= & -\int_{0}^{T}\left(e_{1 t t}, e_{2}\right) d t-\int_{0}^{T}\left(\operatorname{div} r_{1}, e_{2}\right) d t \\
= & -\int_{0}^{T}\left(e_{1 t t}, e_{2}\right) d t-\int_{0}^{T}\left(\operatorname{div} r_{1}, e_{2}\right) d t \\
& +\int_{0}^{T}\left(A^{-1} r_{1}, r_{2}\right) d t-\int_{0}^{T}\left(e_{1}, \operatorname{div} r_{2}\right) d t \\
= & -\int_{0}^{T}\left(e_{2 t t}, e_{1}\right) d t-\int_{0}^{T}\left(\operatorname{div} r_{2}, e_{1}\right) d t \\
& +\int_{0}^{T}\left(A^{-1} r_{2}, r_{1}\right) d t-\int_{0}^{T}\left(e_{2}, \operatorname{div} r_{1}\right) d t \\
= & -\int_{0}^{T}\left(\left(g_{2}^{\prime}\left(y_{h}(u)\right)-g_{2}^{\prime}\left(y_{h}\right), e_{1}\right)\right. \\
& \left.\quad+\left(g_{1}^{\prime}\left(\mathbf{p}_{h}(u)\right)-g_{1}^{\prime}\left(\mathbf{p}_{h}\right), r_{1}\right)\right) d t \\
= & -\int_{0}^{T}\left(\left(g_{2}^{\prime}\left(y_{h}(u)\right)-g_{2}^{\prime}\left(y_{h}\right), y_{h}(u)-y_{h}\right)\right. \\
& \left.+\left(g_{1}^{\prime}\left(\mathbf{p}_{h}(u)\right)-g_{1}^{\prime}\left(\mathbf{p}_{h}\right), \mathbf{p}_{h}(u)-\mathbf{p}_{h}\right)\right) d t
\end{aligned}
$$

$\leq 0$,

where we used the fact that $g_{1}$ and $g_{2}$ are convex functionals. By using (119) and $\varepsilon$-Caunchy inequality,

$$
\begin{aligned}
c \| u- & u_{h} \|_{L^{2}\left(j ; L^{2}\left(\Omega_{U}\right)\right)}^{2} \\
\leq & \int_{0}^{T}\left(z_{h}(u)-z, u-u_{h}\right)_{U} d t \\
& +\int_{0}^{T}\left(j^{\prime}\left(u_{h}\right)-j^{\prime}(u), P_{h} u-u\right)_{U} d t \\
& +\int_{0}^{T}\left(j^{\prime}(u)+z, P_{h} u-u\right)_{U} d t \\
& +\int_{0}^{T}\left(z_{h}(u)-z, P_{h} u-u\right)_{U} d t \\
\leq & C\left\|z_{h}(u)-z\right\|_{L^{2}\left(j ; L^{2}(\Omega)\right)}^{2} \\
& +C \varepsilon\left\|u-u_{h}\right\|_{L^{2}\left(J ; L^{2}\left(\Omega_{U}\right)\right)}^{2} \\
& +C \int_{0}^{T}\left\|j^{\prime}(u)+z\right\|_{k+1, \Omega_{U}}\left\|P_{h} u-u\right\|_{-k-1, \Omega_{U}} d t \\
& +C\left\|P_{h} u-u\right\|_{L^{2}\left(j ; L^{2}\left(\Omega_{U}\right)\right)}^{2} \\
\leq & C\left\|z_{h}(u)-z\right\|_{L^{2}\left(j ; L^{2}(\Omega)\right)}^{2} \\
& +C \varepsilon\left\|u-u_{h}\right\|_{L^{2}\left(j ; L^{2}\left(\Omega_{U}\right)\right)}^{2}+C h_{U}^{2(k+1)},
\end{aligned}
$$

for any small $\varepsilon>0$, where $\left\|P_{h} u-u\right\|_{-k-1, \Omega_{U}} \leq$ $C h^{2(k+1)}\|u\|_{k+1, \Omega_{U}}$ has been used. It is easy to see that

$$
\begin{aligned}
& c \| u-u_{h} \|_{L^{2}\left(J ; L^{2}\left(\Omega_{U}\right)\right)} \\
& \quad \leq C\left\|z_{h}(u)-z\right\|_{L^{2}\left(J ; L^{2}(\Omega)\right)}+C h_{U}^{k+1} \leq C\left(h^{k+1}+h_{U}^{k+1}\right) .
\end{aligned}
$$

From Theorems 5 and 6 and (122), we can obtain that

$$
\begin{aligned}
\| y- & y_{h}\left\|_{L^{\infty}\left(j ; L^{2}(\Omega)\right)}+\right\| \mathbf{p}-\mathbf{p}_{h} \|_{L^{\infty}\left(j ;\left(L^{2}(\Omega)\right)^{2}\right)} \\
& +\left\|z-z_{h}\right\|_{L^{\infty}\left(j ; L^{2}(\Omega)\right)}+\left\|\mathbf{q}-\mathbf{q}_{h}\right\|_{L^{\infty}\left(j ;\left(L^{2}(\Omega)\right)^{2}\right)} \\
\leq & \left\|y-y_{h}(u)\right\|_{L^{\infty}\left(J ; L^{2}(\Omega)\right)}+\left\|\mathbf{p}-\mathbf{p}_{h}(u)\right\|_{L^{\infty}\left(J ;\left(L^{2}(\Omega)\right)^{2}\right)} \\
& +\left\|z-z_{h}(u)\right\|_{L^{\infty}\left(j ; L^{2}(\Omega)\right)} \\
& +\left\|\mathbf{q}-\mathbf{q}_{h}(u)\right\|_{L^{\infty}\left(j ;\left(L^{2}(\Omega)\right)^{2}\right)}+\left\|y_{h}(u)-y_{h}\right\|_{L^{\infty}\left(j ; L^{2}(\Omega)\right)} \\
& +\left\|\mathbf{p}_{h}(u)-\mathbf{p}_{h}\right\|_{L^{\infty}\left(J ;\left(L^{2}(\Omega)\right)^{2}\right)} \\
& +\left\|z_{h}(u)-z_{h}\right\|_{L^{\infty}\left(j ; L^{2}(\Omega)\right)}+\left\|\mathbf{q}_{h}(u)-\mathbf{q}_{h}\right\|_{L^{\infty}\left(J ;\left(L^{2}(\Omega)\right)^{2}\right)} \\
\leq & \left\|y-y_{h}(u)\right\|_{L^{\infty}\left(J ; L^{2}(\Omega)\right)}+\left\|\mathbf{p}-\mathbf{p}_{h}(u)\right\|_{L^{\infty}\left(J ;\left(L^{2}(\Omega)\right)^{2}\right)} \\
& +\left\|\mathbf{q}-\mathbf{q}_{h}(u)\right\|_{L^{\infty}\left(J ;\left(L^{2}(\Omega)\right)^{2}\right)}+\left\|z-z_{h}(u)\right\|_{L^{\infty}\left(j ; L^{2}(\Omega)\right)} \\
& +C\left\|u-u_{h}\right\|_{L^{2}\left(j ; L^{2}(\Omega)\right)} \\
\leq & C\left(h^{k+1}+h_{U}^{k+1}\right) .
\end{aligned}
$$

Then we complete the proof.

\section{Conclusion and Future Works}

In this paper we presented a priori error estimate for mixed finite element approximation of the general linear hyperbolic optimal control problems (1)-(5). Using the elliptic projection operators and Gronwall's Lemma, we have established some error estimate results for both the state and the costate discrete solutions and the control approximation. To the best of our knowledge in the context of optimal control problems, these a priori error estimates for the general hyperbolic optimal control problems are new. In our future work, we will use the fully discrete mixed finite element method to deal with nonlinear hyperbolic optimal control problems. Furthermore, we will consider a priori error estimates and superconvergence of these optimal control problems.

\section{Conflict of Interests}

The authors declare that there is no conflict of interests regarding the publication of this paper. 


\section{Acknowledgments}

The authors express their thanks to the referees for their helpful suggestions, which led to improvements of the presentation. This work is supported by National Science Foundation of China (11201510), Chongqing Research Program of Basic Research and Frontier Technology (cstc2012jjA00003), Scientific and Technological Research Program of Chongqing Municipal Education Commission (KJ121113), and Science and Technology Project of Wanzhou District of Chongqing (2013030050).

\section{References}

[1] R. S. Falk, "Approximation of a class of optimal control problems with order of convergence estimates," Journal of Mathematical Analysis and Applications, vol. 44, pp. 28-47, 1973.

[2] W. Alt and U. Mackenroth, "Convergence of finite element approximations to state constrained convex parabolic boundary control problems," SIAM Journal on Control and Optimization, vol. 27, no. 4, pp. 718-736, 1989.

[3] N. Arada, E. Casas, and F. Tröltzsch, "Error estimates for the numerical approximation of a semilinear elliptic control problem," Computational Optimization and Applications, vol. 23, no. 2, pp. 201-229, 2002.

[4] H. Chen, R. Ewing, and R. Lazarov, "Superconvergence of mixed finite element methods for parabolic problems with nonsmooth initial data," Numerische Mathematik, vol. 78, no. 4, pp. 495-521, 1998.

[5] Y. Chen, Y. Huang, W. Liu, and N. Yan, "Error estimates and superconvergence of mixed finite element methods for convex optimal control problems," Journal of Scientific Computing, vol. 42, no. 3, pp. 382-403, 2010.

[6] Y. Chen and W. Liu, "Error estimates and superconvergence of mixed finite element for quadratic optimal control," International Journal of Numerical Analysis and Modeling, vol. 3, no. 3, pp. 311-321, 2006.

[7] Y. Chen and Z. Lu, "Error estimates of fully discrete mixed finite element methods for semilinear quadratic parabolic optimal control problem," Computer Methods in Applied Mechanics and Engineering, vol. 199, no. 23-24, pp. 1415-1423, 2010.

[8] Y. Chen and Z. Lu, "Error estimates for parabolic optimal control problem by fully discrete mixed finite element methods," Finite Elements in Analysis and Design, vol. 46, no. 11, pp. 957965, 2010.

[9] Y. Chen, Z. Lu, and Y. Huang, "Superconvergence of triangular Raviart-Thomas mixed finite element methods for a bilinear constrained optimal control problem," Computers and Mathematics with Applications, vol. 66, no. 8, pp. 1498-1513, 2013.

[10] G. Knowles, "Finite element approximation of parabolic time optimal control problems," SIAM Journal on Control and Optimization, vol. 20, no. 3, pp. 414-427, 1982.

[11] K. Kunisch, W. Liu, Y. Chang, N. Yan, and R. Li, "Adaptive finite element approximation for a class of parameter estimation problems," Journal of Computational Mathematics, vol. 28, no. 5, pp. 645-675, 2010.

[12] J. L. Lions, Optimal Control of Systems Governed by Partial Differential Equations, Springer, Berlin, Germany, 1971.

[13] W. Liu and N. Yan, "A posteriori error estimates for optimal control problems governed by parabolic equations," Numerische Mathematik, vol. 93, no. 3, pp. 497-521, 2003.
[14] Z. Lu and Y. Chen, "A posteriori error estimates of triangular mixed finite element methods for semilinear optimal control problems," Advances in Applied Mathematics and Mechanics, vol. 1, no. 2, pp. 242-256, 2009.

[15] Z. Lu and Y. Chen, "A posteriori error estimates of lowest order Raviart-Thomas mixed finite element methods for bilinear optimal control problems," Numerical Analysis and Applications, vol. 12, pp. 74-86, 2009.

[16] Z. Lu, Y. Chen, and W. Zheng, "A posteriori error estimates of lowest order Raviart-Thomas mixed finite element methods for bilinear optimal control problems," East Asian Journal on Applied Mathematics, vol. 2, no. 2, pp. 108-125, 2012.

[17] F. Brezzi and M. Fortin, Mixed and Hybrid Finite Element Methods, Springer, Berlin, Germany, 1991.

[18] Y. Chen and W. Liu, "Posteriori error estimates for mixed finite elements of a quadratic optimal control problem," Recent Progress in Computational and Applied PDES, vol. 2, pp. 123-134, 2002.

[19] Y. Chen and W. Liu, "A posteriori error estimates for mixed finite element solutions of convex optimal control problems," Journal of Computational and Applied Mathematics, vol. 211, no. 1, pp. 76-89, 2008.

[20] T. Hou, "A posteriori $\mathrm{L}^{\infty}\left(\mathrm{L}^{2}\right)$-error estimates of semidiscrete mixed finite element methods for hyperbolic optimal control problems," Bulletin of the Korean Mathematical Society, vol. 50, no. 1, pp. 321-341, 2013.

[21] X. Xing and Y. Chen, "Error estimates of mixed methods for optimal control problems governed by parabolic equations," International Journal for Numerical Methods in Engineering, vol. 75, no. 6, pp. 735-754, 2008.

[22] S. M. F. Garcia, "Improved error estimates for mixed finiteelement approximations for nonlinear parabolic equations: the continutious-time case," Numerical Methods for Partial Differential Equations, vol. 28, pp. 129-146, 1993.

[23] D. Gilbarg and N. Trudinger, Elliptic Partial Differential Equations of Second Order, Springer, Berlin, Germany, 1977.

[24] Y. Chen, "Mixed finite element methods for a strongly nonlinear parabolic problem," Journal of Computational Mathematics, vol. 17, no. 2, pp. 209-220, 1999.

[25] F. A. Milner, "Mixed finite element methods for quasilinear second-order elliptic problems," Mathematics of Computation, vol. 44, no. 170, pp. 303-320, 1985.

[26] M. F. Wheeler, "A priori $L_{2}$ error estimates for Galerkin approximations to parabolic partial differential equations," SIAM Journal on Numerical Analysis, vol. 10, pp. 723-759, 1973.

[27] F. A. Miliner and E. J. Park, "A mixed finite element methods for a strongly nonlinear second order elliptic problem," Mathematics of Computation, vol. 211, pp. 973-988, 1995.

[28] L. Evans, Partial Differential Equations, American Mathematical Society, Providence, RI, USA, 1998. 


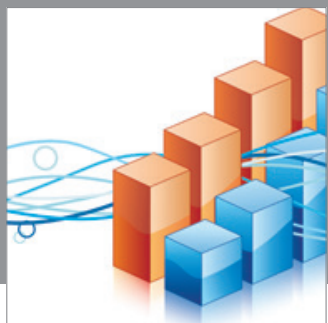

Advances in

Operations Research

mansans

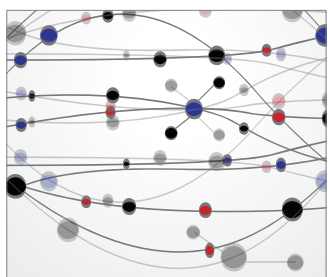

The Scientific World Journal
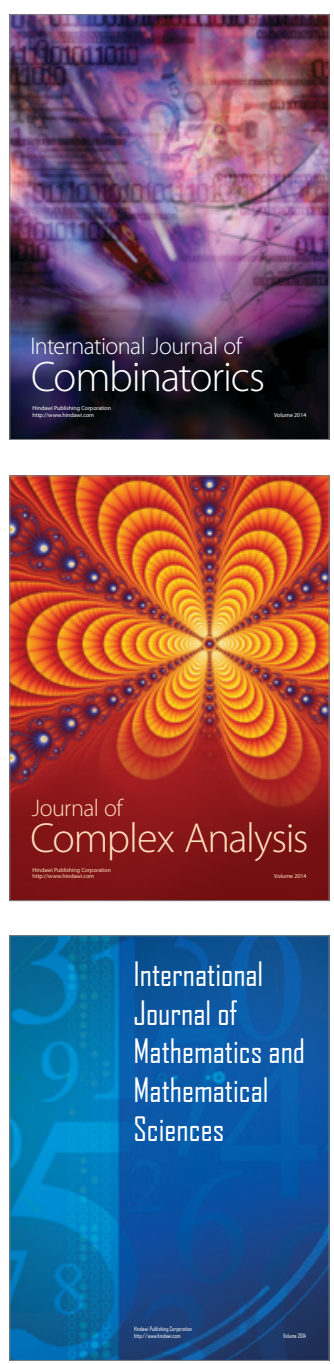
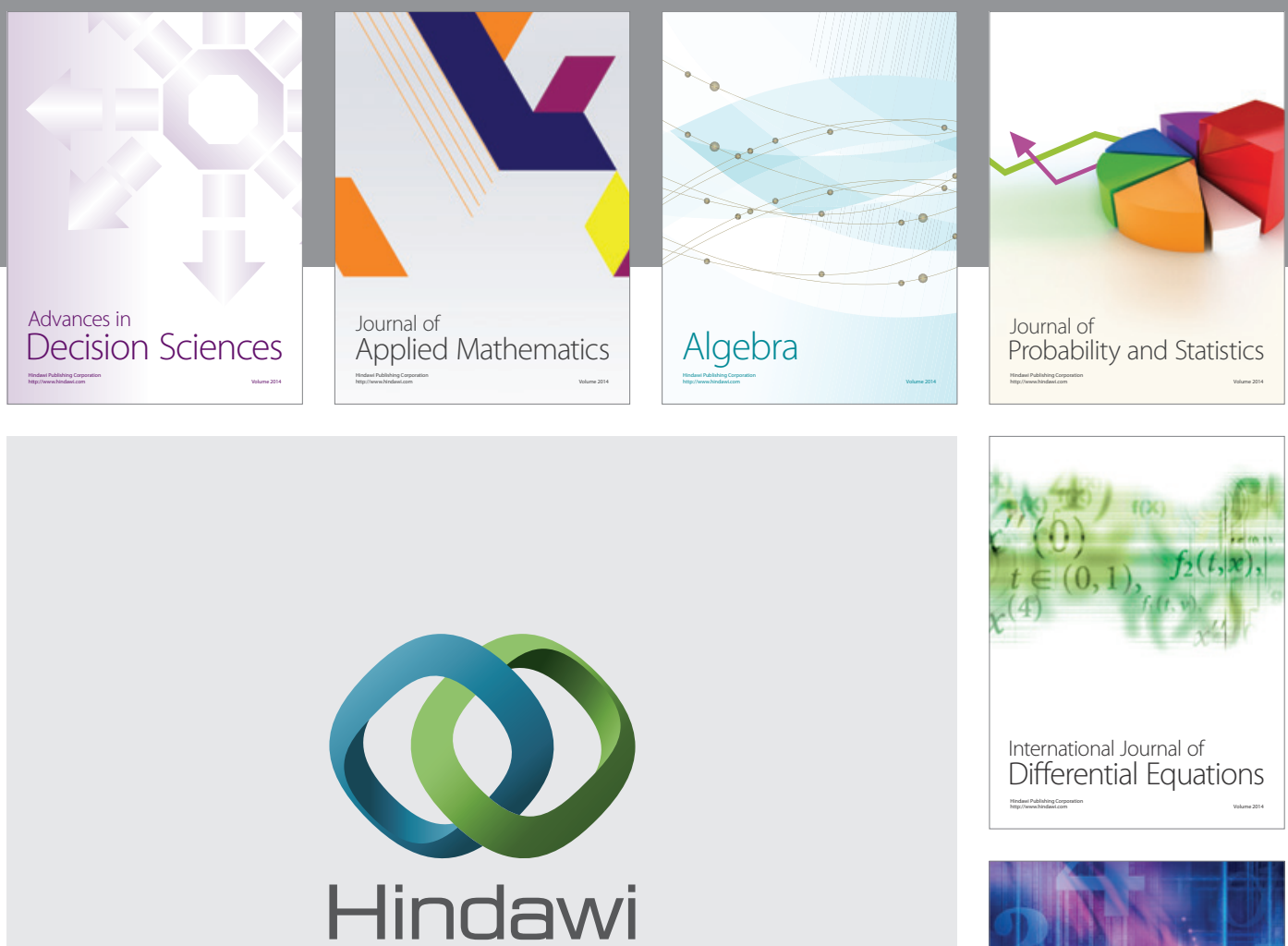

Submit your manuscripts at http://www.hindawi.com
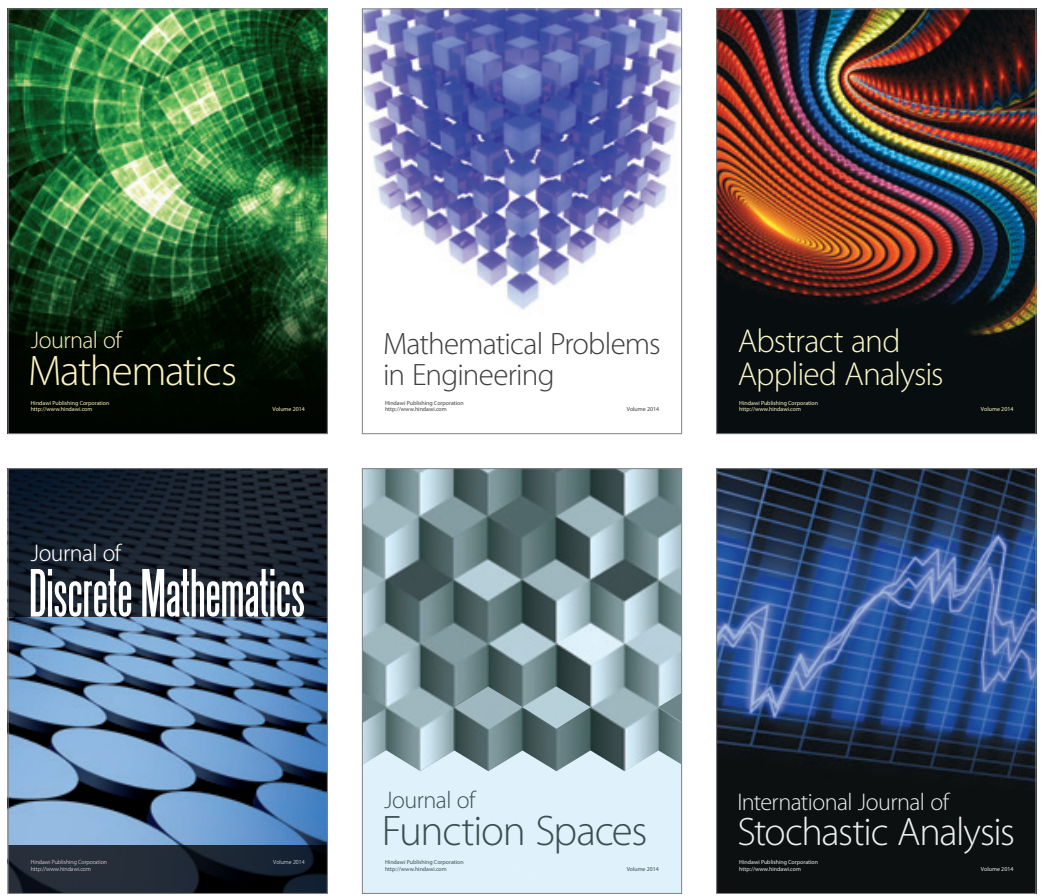

Journal of

Function Spaces

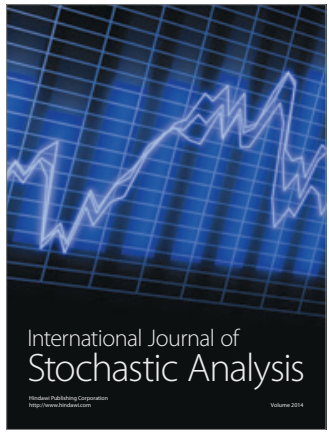

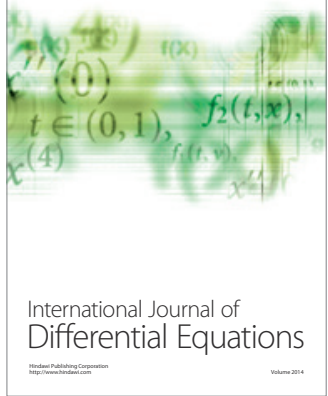
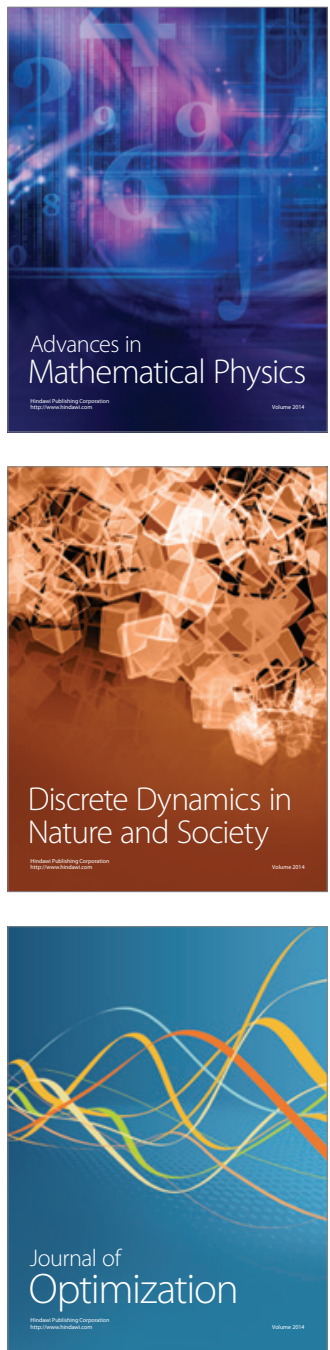